\title{
Dental evaluation of scuba diving mouthpieces using a subject assessment index and radiological analysis of jaw position
}

\author{
R S Hobson, J P Newton
}

\begin{abstract}
Objective-To compare two experimental scuba mouthpieces with a commercially available design.

Methods-A laboratory study using six men to assess effort, muscle pain, muscle fatigue, facial discomfort, tooth discomfort, and loss of lip sensation using a visual analogue scale. Cephalometric radiographs and analysis of jaw position with each mouth piece were also used.

Results-Fully customised mouthpieces caused the least discomfort, muscle pain, fatigue, and effort. They also resulted in the least mandibular displacement from the resting position. Radiographic analysis of jaw position showed that the fully customised design resulted in the least displacement from normal jaw position. Conclusions-A fully customised design gives the greatest comfort, least effort, and least mandibular displacement. This design is recommended, particularly for divers who experience temporomandibular dysfunction associated with diving. (Br f Sports Med 2001;35:84-88)
\end{abstract}

Keywords: scuba diving; temporomandibular dysfunction; mouthpieces; teeth; jaw

The introduction of the self contained underwater breathing apparatus (scuba) by Cousteau and Gagin in the 1940s has enabled underwater diving to develop both commercially and as a sport. Although the design of the demand valve, which regulates the air supply to the diver, has been improved substantially, the mouthpiece by which the diver holds the demand valve in place has undergone little or no development.

Pinto $^{1}$ and Roydhouse ${ }^{2}$ have suggested that the use of a scuba mouthpiece can cause local inflammation of the temporomandibular joint, which can lead to labyrinthine dysfunction and associated vestibular disturbances-for example, vertigo and disorientation. These are potentially hazardous conditions which have been implicated in underwater accidents and deaths. ${ }^{3}$ Other workers ${ }^{2}$ have found that the incidence of temporomandibular joint dysfunction associated with the use of a diving mouthpiece is as high as $65 \%$ of all divers.

The present commercially available mouthpieces are constructed of either rubber or silicone material and all share a similar design. They have an airway tube (fig 1), which connects to the demand valve passing air to the diver, and an oral screen which fits inside the diver's lips aiding retention and providing a seal. The diver bites on two interdental platforms to hold the mouthpiece and demand valve in place, this being aided by palatal lugs on the inside of the teeth. The canine and premolar teeth (fig 2) hold these bite platforms. This basic design appears to have a number of faults which can result in the temporomandibular joint being incorrectly loaded.

Goldstien and $\mathrm{Katz}^{3}$ and Lamendin ${ }^{5}$ have previously attempted to improve the mouthpiece design, although with no reported clinical trials. Mack et al $l^{6}$ reported the outcome of an underwater trial using a number of commercial and experimental designs, concluding that the design parameters were mutually antagonistic but that thin bite platforms placed between the premolar and molar teeth should reduce the incidence of temporomandibular joint dysfunction associated with diving.

In a study of commercially available mouthpieces, using electromyographic recordings

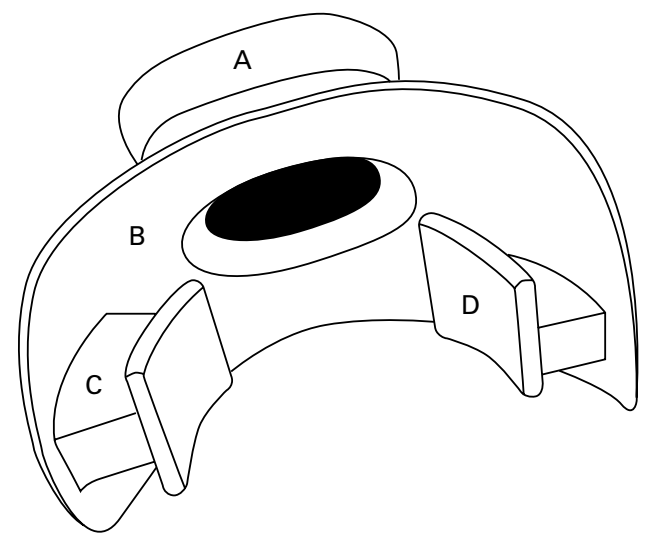

Figure 1 Diagram to show the general form and shape of a scuba mouthpiece. A, Connection to demand valve; $B$, oral screen; $C$, interdental bite platform; $D$, palatal lugs. 


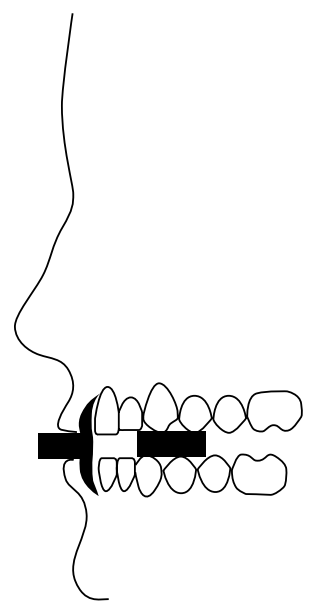

Figure 2 Diagram to show the conventional position of the scuba mouthpiece as it is held between the canine and premolar teeth. Note that the posterior teeth are not in occlusion. from the masseter muscle, Ingervall and Warvfinge $^{7}$ found that a semicustomised design required less muscle activity for retention than commercial designs. We have previously described the development and manufacture of a fully customised scuba mouthpiece, ${ }^{8}$ which was developed to fit the diver's occlusion with the aim of reducing the incidence of temporomandibular joint dysfunction.

The present study compares a commercial design with two experimental designs, one of which was fully customised, using a subject assessment index and radiological analysis of the jaw position during its use in a simulated dive procedure.

\section{Materials and methods}

The study involved six male non-divers, aged between 25 and 35, all of whom had given their consent. They possessed a complete natural dentition, with a class I skeletal pattern and occlusion, and there was no history of temporomandibular joint dysfunction or any discomfort in their masticatory muscles.

Three mouthpiece designs were examined: a commercially available design (Scubapro UK Ltd, Mitcham, Surrey, UK) (fig 3A), a semicustomised design (fig 3B), and a fully customised design (fig 3C). The semicustomised design was produced using the parameters suggested by Mack et $a l,{ }^{6}$ and the fully customised design was produced as previously described $^{8}$ on the casts of the subject's dentition mounted on a dental articulator. The mouthpieces were used in a random sequence with each subject being tested with all three designs. A minimum of at least seven days was allowed to elapse between experimental sessions for each subject, the duration of the experimental period being 45 minutes in every case. Each subject was seated upright in a dental chair with the head supported by a head rest. A scuba diving demand valve (Scubapro Mk V) was attached in the normal position for a dive, and the mouthpiece under investigation was placed in the subject's mouth.

A visual analogue scale questionnaire was prepared for completion by the subject during each experimental session. The following parameters were assessed: effort required to hold the mouthpiece in place; facial discomfort; muscle fatigue; muscle pain; change in lip sensation (numbness); tooth discomfort; any tooth pain. The subjects were asked to complete the self assessment questionnaire at one minute, five minutes, and subsequent five minute intervals for the 45 minute experimental period. Responses were recorded by a single mark on a $10 \mathrm{~cm}$ visual analogue scale, which had extremes indicated at the scale limits-for example, for muscle pain, the limits were "No pain whatsoever" to "More pain than I have ever experienced".

To assess reproducibility, a subject was selected at random and the procedures repeated for all three mouthpieces. No significant differences were found ( $t$ test, $\mathrm{p}>0.05$ ). Analysis of variance was used to examine the difference in the response to the various mouthpieces.
A
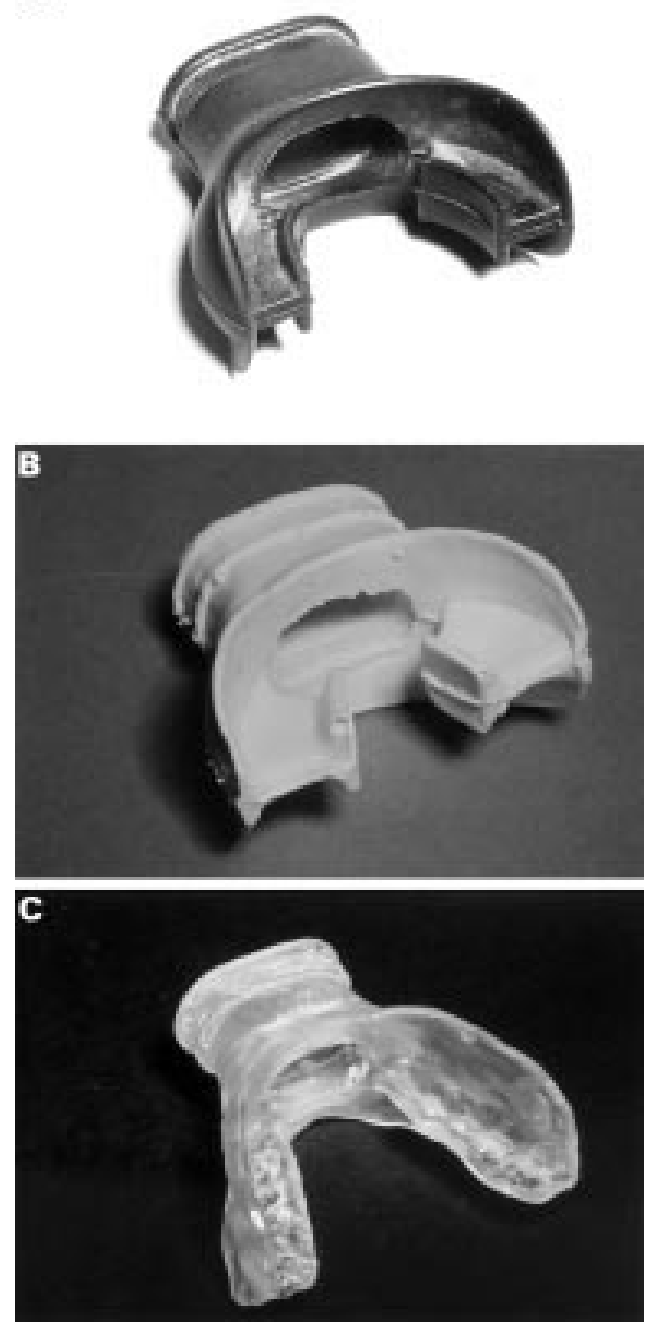

Figure 3 (A) Commercial mouthpiece constructed from rubber or silicone, with the bite blocks positioned to be gripped by the canine and premolar teeth. (B)

Semicustomised mouthpiece constructed from silicone, with thin bite blocks positioned to be gripped by the premolar and molar teeth. (C) Customised mouthpiece constructed from silicone, with thin bite blocks positioned to be gripped by the canine, premolar, and molar teeth. Notice that the bite blocks reflect the cusps of the user's dentition.

Three subjects were selected at random and cephalometric lateral skull radiographs were taken to examine the differences in mandibular position during the use of each mouthpiece. Cephalometric radiography was developed by Broadbent $^{9}$ to interpret lateral skull radiographs taken in a standardised cephalostat. The radiographs were traced and analysed using an orthodontic computer program (COGSoft; British Orthodontic Society, Eastman Dental Hospital, London, UK) on an IBM compatible PC with a digitising tablet, superimposing on the Sella-Nasion line at Sella.

\section{Results}

SUBJECT ASSESSMENT

The visual analogue scales were measured to an accuracy of $1 \mathrm{~mm}$ and the data averaged at each time interval for each mouthpiece. Mean scores for each of the three mouthpieces are given for effort (fig 4), muscle pain (fig 5), 


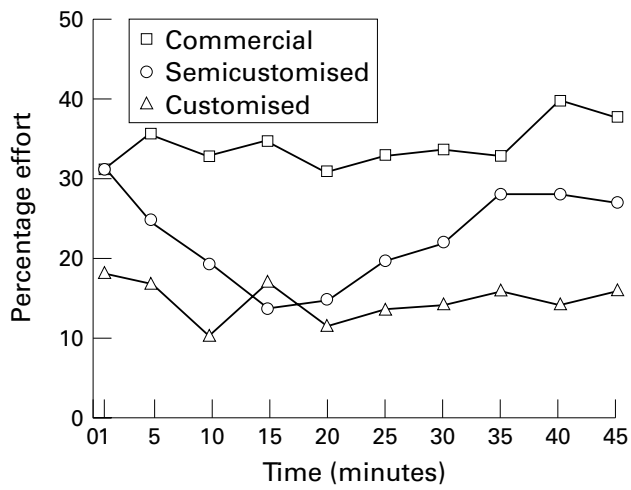

Figure 4 Plot of perceived effort to hold mouthpieces in place against time.

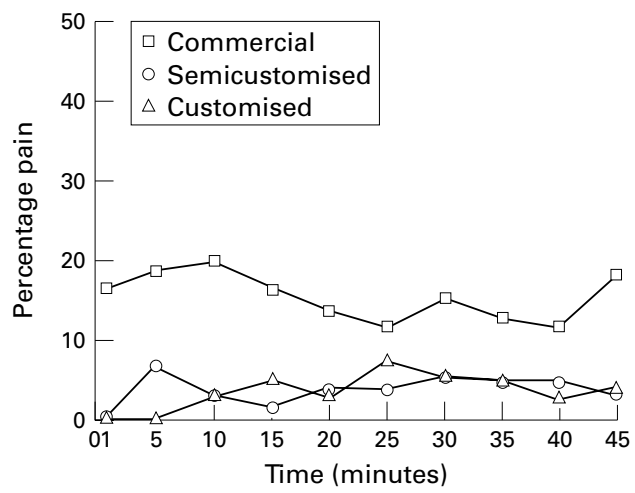

Figure 5 Plot of perceived muscle pain during use of mouthpieces against time.

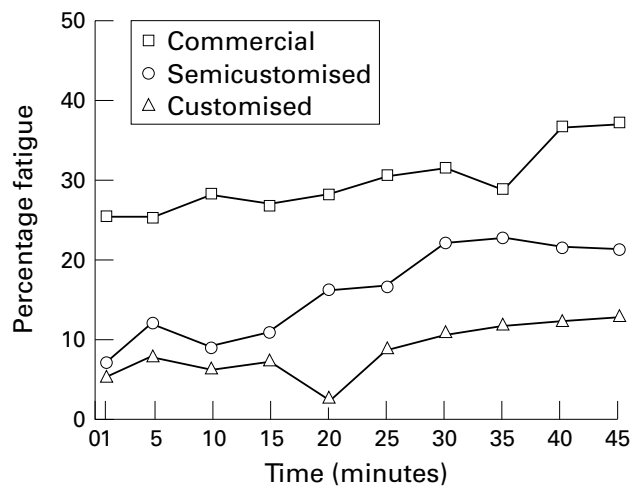

Figure 6 Plot of perceived muscle fatigue during use of mouthpieces against time.

muscle fatigue (fig 6), facial discomfort (fig 7), loss of lip sensation (fig 8), and tooth discomfort (fig 9). No tooth pain was reported at all with any of the mouthpieces.

The greatest effort was required to hold the commercial mouthpiece in place (34\% effort), compared with $22 \%$ for the semicustomised mouthpiece $(p<0.001)$ and $15 \%$ for the customised mouthpiece $(p<0.001)$. The effort to hold the customised mouthpiece in place was significantly less than that required for the semicustomised mouthpiece $(\mathrm{p}<0.005)$. It was noticeable that the effort to hold the mouthpiece increased as time passed for both the commercial and semicustomised designs, but not for the fully customised design.

Figure 6 shows that, as expected from the effort to hold the mouthpiece, there was a gradual increase in muscle fatigue with time

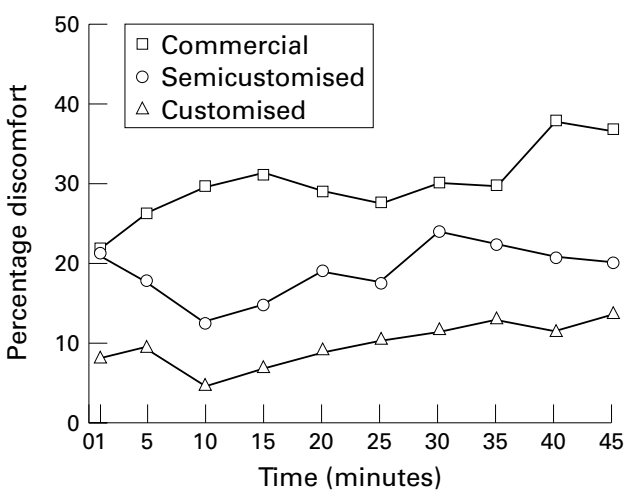

Figure 7 Plot of perceived facial discomfort during use of mouthpieces against time.

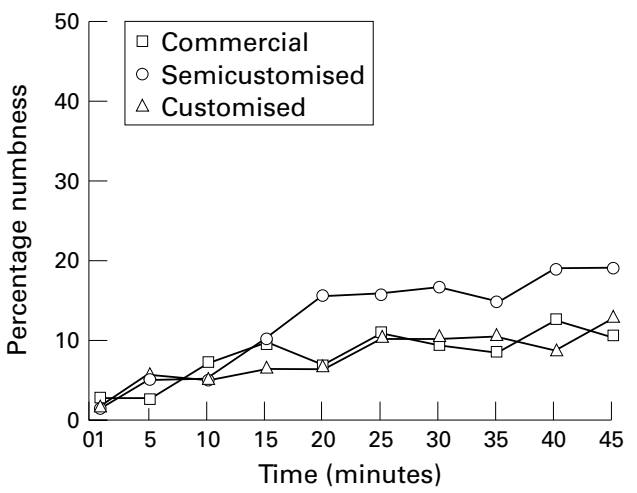

Figure 8 Plot of perceived loss of lip sensation during use of mouthpieces against time.

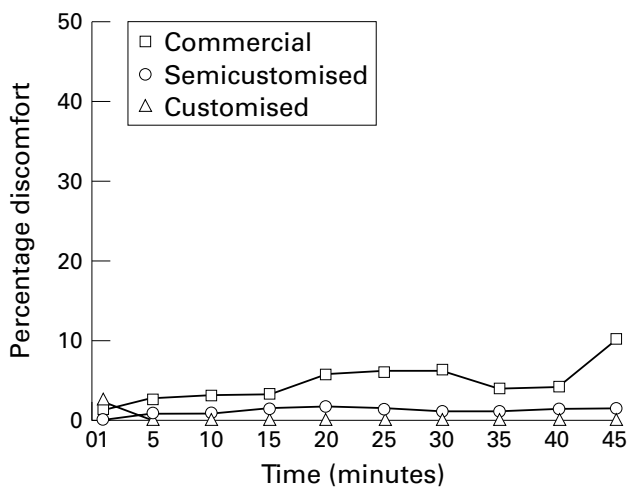

Figure 9 Plot of perceived tooth discomfort during use of mouthpieces against time.

with all three mouthpieces. The commercial design caused the greatest discomfort when compared with both the semicustomised and customised design $(p<0.001)$.

Although, the amount of muscle pain experienced was low for all three designs (fig 5), the commercial design caused almost twice as much pain as the semicustomised and customised designs $(p<0.005)$. Figure 7 shows the overall discomfort resulting from the use of the mouthpieces and a similar increasing trend in discomfort is observed. The commercial design produced the most discomfort followed by the semicustomised design, with the customised design causing the least $(p<0.001)$.

Discomfort to the dentition was very low (fig 9), and, although there was a significant difference between that caused by the commercial design and the semicustomised and customised 


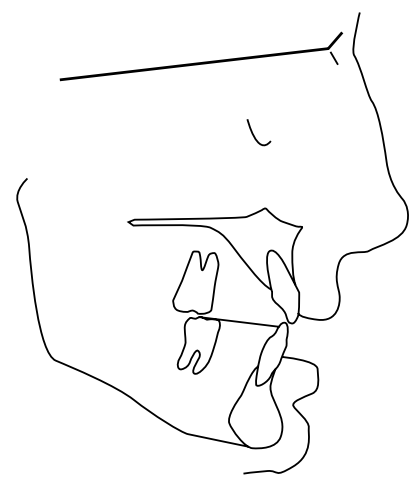

Normal occlusion

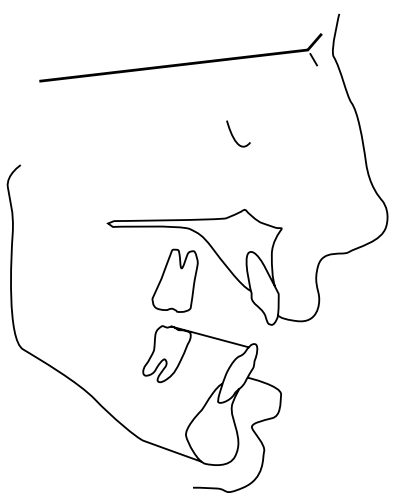

Semicustomised mouthpiece

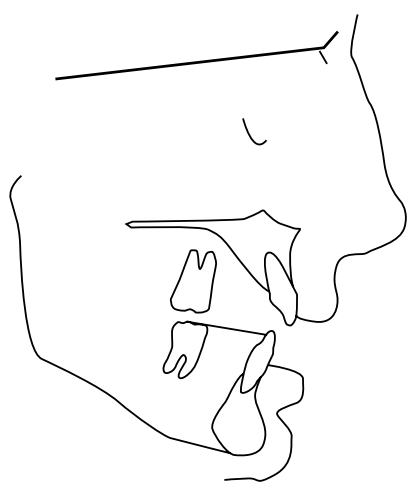

Customised mouthpiece

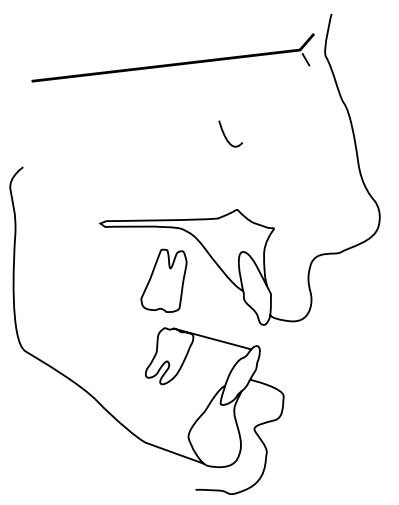

Commercial mouthpiece
Figure 10 Diagram showing the average differences in mandibular position during mouthpiece use. Normal occlusion is shown for comparison.

designs $(\mathrm{p}<0.05)$, this is probably not clinically significant. The extent of loss of sensation (fig 8 ), perceived as the lips feeling numb, showed a gradual increase with time. The commercial, semicustomised, and customised mouthpieces had no perceivable difference between them $(p>0.8)$. However, there was a trend indicating that the commercial mouthpiece produced the greatest change when compared with the other designs.

None of the subjects reported temporomandibular joint dysfunction either during or after the experimental period. Subsequent follow up one week and four weeks later confirmed this observation.

CEPHALOMETRIC RADIOGRAPHS

Mean cephalometric tracings in fig 10 show the extent of displacement by the three different mouthpieces in comparison with normal occlusion. Table 1 gives the mean changes in cephalometric values. The greatest forward displacement of the mandible was produced by the

Table 1 Mean change between normal mandibular position and mandibular position with mouthpieces in situ

\begin{tabular}{llll}
\hline & Customised & Semicustomised & Commercial \\
\hline SNB angle $\left(^{\circ}\right)$ & -0.5 & 0.0 & +3 \\
Mx/Mn angle $\left(^{\circ}\right)$ & +2 & +4 & +4.5 \\
Lower face height $(\mathrm{mm})$ & +3 & +6 & +6 \\
Overjet $(\mathrm{mm})$ & 0.0 & -1.0 & -3 \\
Overbite $(\mathrm{mm})$ & +1 & +5.0 & +5.5 \\
\hline
\end{tabular}

$\mathrm{Mx} / \mathrm{Mn}$ angle, angle between the maxilla and mandible. commercial design. This is confirmed by the increase in the Sella-Nasion-B point (SNB) angle, which measures the position of the mandible in relation to the anterior cranial base. The largest change was caused by the commercial mouthpiece, the customised and semicustomised mouthpieces having a minimal effect on mandibular displacement. The overjet, the horizontal measurement of the upper to the lower incisor teeth, confirms the forward displacement caused by the commercial mouthpiece, with a reduction - that is, forward movement of lower incisors-of $-3 \mathrm{~mm}$.

The mandible is also displaced downwards, rotating backwards around the temporomandibular joint, by all mouthpieces, with the semicustomised and commercial mouthpieces causing the biggest increase in the angle between the maxilla and mandible. The reduced face height (a measure of vertical displacement of the mandible) and the increase in overbite (the vertical distance between the upper and lower incisors) confirm that these mouthpieces displace the mandible downwards.

In all three subjects during use of the customised mouthpiece, the mandibular position was close to that of the normal rest position, with the teeth slightly apart, producing the minimum displacement.

\section{Discussion}

The experimental method used in this study does not truly reflect the use of the scuba diving mouthpiece in a diving environment, as the diver normally swims in a prone face down position with the head extended. Obviously, it was difficult to reproduce this in the laboratory, but the upright position should provide similar loading of the mouthpiece by the demand valve. In addition, it was not possible to take into account the affect of stress, both psychological and physical, that occurs during an underwater dive, which may affect the force exerted by the diver on the mouthpiece. It is known that parafunctional activity of the masticatory apparatus, such as clenching, can occur in response to stress ${ }^{10}$ or as a result of exposure to cold. Nevertheless, the results confirm other clinical findings that a semicustomised mouthpiece requires significantly less muscular effort for its retention than a commercial mouthpiece. ${ }^{67}$

Ranking of the mouthpieces using the subject assessment index supports the findings of Mack et $a l^{7}$ that a semicustomised design can reduce the perceived muscle effort, fatigue, and discomfort compared with the commercially available design. However, the fully customised mouthpiece shows a significant improvement over both of these and could be considered the one of choice. This result could have been expected because the other two mouthpieces significantly obliterate the interocclusal rest space of the user. In previous studies of patients who have undergone restorative treatment of their dentition or have been provided with complete dentures or bite raising appliances, 
consequent fundamental decreases in the interocclusal rest space have often led to discomfort in the temporomandibular joint and/or the masticatory muscles. ${ }^{11}$ In the present study, the postural face height of the subjects was determined and the interocclusal rest space was found to be between 3 and $6 \mathrm{~mm}$.

The effect of the different mouthpieces on the perceived changes in lip sensation is a previously unreported finding, and discussion with active divers confirmed that this sensation of numbness often occurs during diving procedures. However, they all had previously attributed this change in sensation to the cold water temperature experienced during the dive and not the use of a diving mouthpiece. Interestingly, Ingervall and Warfvinge ${ }^{7}$ reported a decrease in lip muscle activity with the use of a mouthpiece that achieved a better jaw relationship than two other commercially available designs. This is obviously an area that requires further investigation to elucidate the full role of circum-oral musculature in the retention of diving mouthpieces of different designs.

Cephalometric analysis showed that the fully customised design produced the least protrusion and lowering of the mandible, followed by the semicustomised and commercial designs. The fully customised mouthpiece was originally designed to replicate the rest position of the mandible, and this appears to have been achieved. This also resulted in minimum effort to retain the mouthpiece with a consequent significant reduction in discomfort during its use. It is interesting to note that Ingervall and Warvfinge $^{7}$ found that the highest levels of muscle activity were required for the mouthpiece that produced the least protrusion and lowering of the mandible.

The results from this study suggest that the trend in scuba diving mouthpiece design should be towards an increased level of customisation. In addition, the following design features, which have been shown to have beneficial outcomes for the diver in the short and long term, should be included.
- The bite platforms should extend between the premolar and molar teeth.

- The oral screen should extend fully into the sulcus of the mouth incorporating the labial and buccal aspects.

- The thickness of the bite platforms should be such that the interocclusal rest space (the distance between the intercuspal position and the postural position) is not exceeded.

There are some practical implications, which should be carefully considered at this stage. There are potential problems in the construction process because of the number of stages involved and obviously the greater expense. Furthermore, experienced divers are likely to be reluctant to change from the standard commercial mouthpiece, even though a field trial of the fully customised design by Roberts ${ }^{12}$ produced favourable results. However, the use of a customised mouthpiece should be seriously considered by divers who have previously had episodes of temporomandibular joint dysfunction or prolonged discomfort in their jaw muscles following diving activities.

1 Pinto OF. Temporomandibular joint problems in underwater activities. Fournal of Prosthetic Dentistry 1966;16:772-81.

2 Roydhouse N. The jaw and scuba diving. Fournal of the Otolaryngological Society of Australia 1977;4:162-5.

3 Goldstien GR, Katz W. Diver's mouth syndrome. N Y State Dent F 1982;10:523-5.

4 Hobson RS. Temporomandibular dysfunction syndrome associated with scuba diving mouthpieces. Brf Sports Med 1991;25:49-51.

5 Lamendin H. Pour la plongee sub-aquatique: de la personnalisation dlembout standard a l'embout personalise. Le Chirurgien Dentiste de France 1985;312:45-7.

6 Mack PJ, Hobson RS, Askell J. Dental factors in scuba mouthpiece design. Br Dent f 1987;158:141-2.

7 Ingervall $\mathrm{B}$, Warfvinge J. Activity of orofacial musculature during use of mouthpieces for diving. F Oral Rehabil 1978; during use of

8 Newton JP, Hobson RS, Sturrock KC. The design and construction of customized mouthpieces for scuba diving. Eur fournal of Prosthetic Restoration Dentistry 1995;3:223-6.

9 Broadbent BH. A new X-ray technique and its application to orthodontics. Angle Orthodontist 1931;1:45-66.

10 Lewis MAO, Lamey P-J. Psychological disease. In: Clinical oral medicine. Oxford: Wright, 1993.

11 Yemm R. Analysis of patients referred over a period of five years to a teaching hospital consultant service in dental years to a teaching hospital consultant
prosthetics. Brit Dent $\mathcal{F} 1985 ; \mathbf{1 5 9}: 304-6$.

12 Roberts GV. Divers mouth syndrome: a field study. Dental Update 2000;27:74-7.

\section{Take home message}

Commercially available scuba mouthpiece designs can result in temporomandibular joint and jaw muscle problems. Symptoms can be significantly reduced by the use of a fully customised mouthpiece. 